\title{
VEGF localisation in diabetic retinopathy
}

\author{
Mike Boulton, David Foreman, Gillian Williams, David McLeod
}

\begin{abstract}
Aim-To determine the staining pattern of vascular endothelial growth factor (VEGF) at different stages of diabetic retinopathy (including post-laser photocoagulation) and to compare staining in excised fibrovascular and fibrocellular (non-diabetic) preretinal membranes.
\end{abstract}

Methods-Immunohistochemical localisation of VEGF, using antibodies raised against $\mathrm{VEGF}_{165}$ and $\mathrm{VEGF}_{121,165,189}$, was carried out on specimens of normal human retina $(n=15)$, diabetic retinas $((a)$ with no overt retinopathy $(n=19)$, (b) with intraretinal vascular abnormalities but no proliferative retinopathy $(n=6)$, (c) with active proliferative retinopathy $(n=6),(d)$ with no residual proliferative retinopathy after photocoagulation therapy $(n=15)$ ), excised diabetic fibrovascular membranes $(n=19)$, and non-diabetic fibrocellular membranes $(n=7)$. The degree and pattern of immunostaining was recorded.

Results-In general, VEGF was absent from the majority of normal retinas. VEGF staining was apparent in most diabetic tissues but the staining pattern was dependent on both the specificity of the antibody used and the category of tissue. Staining with the VEGF $_{165}$ antibody was generally confined to endothelial cells and perivascular regions while the VEGF $_{121,165,189}$ antibody was also associated with extravascular components of the inner retina. Intensity of immunostaining of diabetic eyes was dependent on the severity of retinopathy being least in diabetics with no overt retinopathy and greatest in retinas with proliferative retinopathy. Interestingly, the intensity of immunostaining in diabetic retinas which had undergone laser surgery for proliferative retinopathy was reduced to basal levels. Moderate to intense immunostaining was observed in all fibrovascular and fibrocellular membranes examined.

Department of

Ophthalmology,

Manchester Royal Eye

Hospital, Oxford Road,

Manchester M13 9WH

M Boulton

D Foreman

G Williams

D McLeod

Correspondence to:

Dr Mike Boulton, University Department of

Ophthalmology, Manchester Royal Eye Hospital, Oxford Road, Manchester M13 9WH.

Accepted for publication 19 November 1997

Conclusions-This study supports a circumstantial role for VEGF in the pathogenesis of both the preclinical and proliferative stages of diabetic retinopathy. (Br F Ophthalmol 1998;82:561-568)

Among the microvascular manifestations of diabetic retinopathy are pericyte dropout, basement membrane thickening, microaneurysm formation, and capillary non-perfusion resulting in inner retinal ischaemia and hypoxia. ${ }^{1}$ The precise stimulus for the subsequent preretinal neovascularisation that characterises proliferative diabetic retinopathy
(PDR) remains uncertain, but historically retinal hypoxia has been proposed as the stimulus for release of a diffusible angiogenic factor which promotes neovascularisation from adjacent retinal vessels and rubeosis. ${ }^{2}$ While a number of candidate molecules have been suggested for this role, including basic fibroblast growth factor (bFGF), ${ }^{3}$ platelet derived growth factor (PDGF), ${ }^{4}$ and insulin-like growth factor-1 (IGF-1), ${ }^{5}$ recent evidence has supported vascular endothelial growth factor (VEGF) as an important modulator of PDR.

VEGF is a mitogen for endothelial cells whose expression both in vivo and in vitro can be induced by hypoxia. ${ }^{6}$ Reports have demonstrated high affinity, membrane bound VEGF receptors located on vascular endothelial cells ${ }^{7-9}$ and recently on retinal pigment epithelial cells. ${ }^{10}$ These receptors, which are autophosphorylating tyrosine kinases, are also upregulated by hypoxia. ${ }^{11}{ }^{12}$ In situ hybridisations against VEGF mRNA have shown increased retinal expression in human vasoproliferative retinopathies and also in animal models of ocular neovascularisation. ${ }^{13-17}$ Furthermore, VEGF levels are elevated in vitreous samples from patients with active PDR when compared with (a) diabetic eyes without retinopathy, (b) diabetic eyes with background retinopathy, ${ }^{18-20}$ and (c) diabetic eyes with quiescent PDR and after successful laser photocoagulation. ${ }^{18}$ The aim of this study was to compare the pattern and intensity of VEGF immunostaining in (a) normal human retina, (b) diabetic retina (with and without non-proliferative retinopathy or PDR), (c) laser photocoagulated diabetic retina with presumed neovascular regression, and (d) surgically excised diabetic preretinal membranes, to determine whether there is a correlation between VEGF protein distribution and PDR. In addition, fibrocellular membranes were included as a comparative control for the fibrovascular membranes and two antibodies with differing specificities for VEGF were tested.

\section{Materials and methods}

DONOR EYES

Donor human eyes, fixed in $10 \%$ neutral buffered formalin (NBF) within 12 hours post mortem, were provided by the National Disease Research Interchange (NDRI), Philadelphia, USA. The anterior segment was removed and biomicroscopy of the posterior segment performed (a) to note overt features of retinopathy (for example, preretinal membranes, cotton wool spots, haemorrhages) and (b) to determine the extent of any scatter photocoagulation. Eyes were categorised as follows:

Normal-15 human eyes with no known ophthalmic disease and no history of diabetes. 
Donor age ranged from 20 to 92 years (mean 56 years).

Diabetic with no overt retinopathy - 19 human eyes from diabetics with no clinical history of PDR and no overt features of retinopathy or retinal photocoagulation. Donor age ranged from 44 to 89 years (mean 74 years). A complete medical history was unavailable for all donors but, where available (7/18), the duration of diabetes was between 6 and 25 years.

Diabetic with obvious intraretinal vascular changes but no evidence of PDR - six human eyes from diabetics with no clinical history of PDR and no overt features of PDR or retinal photocoagulation. Retinas exhibited cotton wool spots, haemorrhages, and/or obvious microaneurysms. Donor age ranged from 55 to 96 years (mean 71 years). A complete medical history was unavailable for all donors but, where available $(3 / 6)$, the duration of diabetes was between 3 and 21 years.

Diabetic with $P D R$ - six human eyes from diabetics defined clinically as having PDR and exhibiting preretinal membranes. Donor age ranged from 37 to 76 years (mean 58 years). Duration of diabetes ranged from 3 to 18 years (mean 9 years).

Diabetic with scatter laser photocoagulation but no evidence of active PDR - 15 human eyes from diabetics specified clinically as having had PDR and having received scatter laser photocoagulation (no details were available as to time post laser). No preretinal membranes were observed. Donor age ranged from 41 to 82 years (mean 63 years). Duration of diabetes ranged from 3 to 35 years (mean 17 years).

METHODS

The posterior segment of each eye was cut in the sagittal plane through the centre of the optic nerve head. Cuts were then made perpendicular to this line (a) on the horizontal midline on the nasal side and (b) two cuts were made at approximately $5 \mathrm{~mm}$ above and below the midline on the temporal side. A final vertical cut was made parallel to the initial cut and approximately $3 \mathrm{~mm}$ lateral to the macula. Five $\mu \mathrm{m}$ sections were cut from a portion of retina/ choroid/sclera (a) approximately $3 \mathrm{~mm}$ lateral to the macula and perpendicular to the horizontal plane (this region was chosen owing to its susceptibility to retinal changes associated with diabetes) and (b) other representative areas across the retina (for example, areas of neovascularisation).

FIBROVASCULAR MEMBRANES

Nineteen fibrovascular preretinal membranes were obtained from 19 eyes during closed microsurgery for sequelae of PDR at the Manchester Royal Eye Hospital. Membranes were fixed in $10 \%$ NBF immediately upon removal for a minimum of 12 hours before paraffin wax embedding.

FIBROCELLULAR MEMBRANES

Seven non-vascularised epiretinal membranes were obtained from seven eyes of non-diabetic patients during closed microsurgery for elimi- nation of retinal traction at the Manchester Royal Eye Hospital. Membranes were fixed in $10 \%$ NBF immediately upon removal for a minimum of 12 hours before paraffin wax embedding.

\section{IMMUNOHISTOCHEMISTRY}

Five $\mu \mathrm{m}$ sections $(1-1.3 \mathrm{~cm}$ in length in the case of retinal specimens) were cut and mounted on APES coated slides. Deparaffinised sections were digested with $0.01 \%$ chymotrypsin for 20 minutes at $37^{\circ} \mathrm{C}$. The sections were incubated in $10 \%$ milk proteins (Marvel)/ $10 \%$ normal rabbit serum (Dako) in TRIS buffered saline (TBS) for 60 minutes at room temperature. Excess blocking solution was removed and the sections incubated overnight at $4^{\circ} \mathrm{C}$ in either (a) polyclonal goat anti-human $\mathrm{VEGF}_{165}$ (R\&D Systems) raised against purified insect cell line $S f 21$ derived recombinant human $\mathrm{VEGF}_{165}$ or (b) polyclonal goat anti-

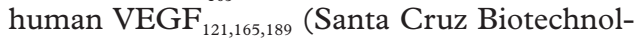
ogy) raised against a peptide corresponding to amino acids 1-20 mapping at the amino terminus of VEGF of human origin and which recognises the 121, 165, and 189 amino acid splice variants of VEGF; both were diluted in TBS to $5 \mu \mathrm{g} / \mathrm{ml}$. Following two 3 minute washes, the sections were incubated with biotinylated rabbit anti-goat IgG (Dako) diluted to 1/600 in TBS for 30 minutes at room temperature. The sections were subsequently incubated with an avidin-biotin alkaline phosphatase reaction complex (Dako) and antibody binding visualised by incubation in fast red substrate solution (Sigma) resulting in the formation of a red product. Immunostained sections were counterstained with haematoxylin.

CONTROLS FOR IMMUNOSTAINING

Negative controls included (1) omission of the primary antibody, (2) substitution of the primary antibody with an inappropriate goat antibody (goat anti-human colostrum whey (Sigma) at the same concentration as the primary antibody), and (3) incubation of 100 $\mu \mathrm{l}(0.5 \mu \mathrm{g})$ of anti-VEGF ${ }_{165}$ antibody with $1 \mu \mathrm{g}$ of recombinant human $\mathrm{VEGF}_{165}$ (R\&D systems) overnight at $4^{\circ} \mathrm{C}$ before use in the above protocol.

ASSESSMENT OF IMMUNOSTAINING

The degree and pattern of immunostaining both within and between specimens as observed by standard light microscopy was assessed and recorded by two independent observers but pathological status of the specimens was obvious. The intensity of staining was graded qualitatively as background (corresponding to the level of staining seen in the negative controls), weak, moderate, or intense (corresponding to the highest level of immunoreactivity observed). These intensities were recorded as $0,1,2$, and 3 respectively. For each retinal specimen staining intensity was recorded for the choroid, RPE, photoreceptor inner and outer segments, outer nuclear layer, inner nuclear layer, retinal vessels, ganglion cell layer, and internal limiting membrane. For 
fibrovascular and fibrocellular membranes staining intensity was recorded for vessels and surrounding matrix.

\section{Results}

VEGF staining was apparent in most diabetic tissue but the staining pattern was dependent on both the specificity of the antibody used and the category of tissue (see Figs 1 and 2 and Tables 1 and 2). Staining with the antiVEGF $_{165}$ antibody was generally confined to endothelial cells (whether retinal or choroidal) and perivascular regions whereas staining with the $\mathrm{VEGF}_{121,165,189}$ antibody was also associated with non-vascular components of the inner retina. No correlation was found between staining intensity/distribution and either donor age, postmortem time, or duration of diabetes (where known).

NORMAL

Immunostaining with the anti-VEGF ${ }_{121,165,189}$ antibody was absent in the majority of retinas examined while retinal staining with the anti-VEGF ${ }_{165}$ antibody, although generally absent, did result in weak staining associated with retinal vessels (7/15) and in the RPE (3/15) (Fig 1A; Tables 1 and 2). Weak to moderate staining with the anti-VEGF $\mathrm{VF}_{165}$ antibody was also observed in the choroidal vessels (12/15). Staining was absent from the inner and outer retina in all but one specimen.

DIABETIC WITH NO OVERT RETINOPATHY

Staining intensity with the anti-VEGF ${ }_{165}$ antibody was not elevated above that observed in non-diabetic retinas. Twelve of 19 specimens exhibited some positive staining of endothelial cells of inner retinal vessels, while 9/19 were VEGF positive in vessel basement membranes (generally those diabetic retinas with thickened endothelial basement membranes). By contrast, immunostaining with the antiVEGF $_{121,165,189}$ antibody, albeit weak to moderate, was elevated in all tissue layers examined compared with that observed in normal retinas (Fig 1B; Table 2). Immunostaining was most intense in the inner retinal and ganglion cell layers (13/19).

DIABETIC WITH OBVIOUS INTRARETINAL

VASCULAR CHANGES BUT NO EVIDENCE OF PDR

Intensity of immunostaining with the anti$\mathrm{VEGF}_{165}$ antibody was increased in the retinal vessels, the choroid, and the ganglion cell layer compared with that observed in normal retinas and diabetic retinas with no overt retinopathy (staining in the other retinal layers remained unchanged) (Figs 1C and 2A). Variable immunostaining ranging from weak to intense was observed in both the retinal vessels and choroid with a close correlation between intense choroidal staining and moderate to intense staining around retinal vessels. Intensity of immunostaining with the anti-VEGF ${ }_{121,165,189}$ antibody in this group was elevated compared with that observed in non-diabetic retinas but not increased compared with that observed for diabetic retina with no overt retinopathy (Fig 1D).

\section{DIABETIC WITH PDR}

Intense immunostaining with the anti-VEGF ${ }_{165}$ antibody of the inner retinal vessels was seen in all diabetic retinas (6/6) with active neovascular PDR membranes on their surfaces (Fig 1E). Equally intense staining was observed within the membranes themselves (Fig 1E). While immunoreactivity for VEGF was found around choroidal vessels in all these PDR retinas, with staining ranging from weak to intense, the RPE layer and outer retina were generally $\mathrm{VEGF}_{165}$ negative. The immunostaining pattern with the anti-VEGF ${ }_{121,165,189}$ antibody showed a similar moderate to high intensity staining of retinal vessels but, in addition, there was increased staining in the outer nuclear layer, inner retina, ganglion cell layer, and internal limiting membrane (ILM) compared with the other tissue groups (Fig 1F).

Table 1 Mean (SD) intensity of immunostaining of the retina/choroid using an anti-VEGF $F_{165}$ antibody

\begin{tabular}{|c|c|c|c|c|c|c|c|}
\hline \multirow[b]{2}{*}{ Tissue category } & \multicolumn{7}{|l|}{ Tissue layer } \\
\hline & $\begin{array}{l}\text { Choroidal } \\
\text { vessels }\end{array}$ & $R P E$ & Outer retina & Inner retina & $\begin{array}{l}\text { Retinal } \\
\text { vessels }\end{array}$ & $\begin{array}{l}\text { Ganglion cell } \\
\text { layer }\end{array}$ & $I L M$ \\
\hline $\begin{array}{l}\text { Normal }(n=12) \\
\text { Diabetic. }\end{array}$ & $1.6(0.3)$ & $0.3(0.2)$ & 0 & $0.3(0.2)$ & $0.9(0.2)$ & $0.1(0.1)$ & 0 \\
\hline \multicolumn{8}{|l|}{ Diabetic: } \\
\hline No overt retinopathy $(\mathrm{n}=19)$ & $1.6(0.2)$ & $0.1(0.1)$ & 0 & $0.4(0.2)$ & $1.2(0.2)$ & $0.2(0.1)$ & $0.1(0.1)$ \\
\hline Vascular abnormalities $(n=6)$ & $2.3(0.3)$ & $0.5(0.3)$ & $0.2(0.2)$ & $0.3(0.2)$ & $2.2(0.5)$ & $1.0(0.4)$ & $0.3(0.2)$ \\
\hline $\operatorname{PDR}(n=6)$ & $2.2(0.4)$ & $0.4(0.3)$ & $0.6(0.2)$ & $0.6(0.2)$ & $3.0(0)$ & $0.6(0.2)$ & $0.6(0.2)$ \\
\hline Laser, no PDR $(n=15)$ & $1.7(0.3)$ & $0.1(0.1)$ & $0.3(0.1)$ & $0.3(0.1)$ & $1.0(0.3)$ & $0.2(0.1)$ & $0.1(0.1)$ \\
\hline
\end{tabular}

$0=$ background, 1 = weak, 2 = moderate, $3=$ intense.

Table 2 Mean (SD) intensity of immunostaining of the retina/choroid using an anti-VEGF ${ }_{121,165,189}$ antibody

\begin{tabular}{|c|c|c|c|c|c|c|c|}
\hline \multirow[b]{2}{*}{ Tissue category } & \multicolumn{7}{|c|}{ Tissue layer } \\
\hline & $\begin{array}{l}\text { Choroidal } \\
\text { vessels }\end{array}$ & $R P E$ & Outer retina & Inner retina & $\begin{array}{l}\text { Retinal } \\
\text { vessels }\end{array}$ & $\begin{array}{l}\text { Ganglion cell } \\
\text { layer }\end{array}$ & $I L M$ \\
\hline Normal $(n=12)$ & $0.3(0.1)$ & $0.5(0.4)$ & $0.2(0.1)$ & $0.2(0.1)$ & $0.3(0.1)$ & $0.4(0.2)$ & $0.3(0.2)$ \\
\hline \multicolumn{8}{|l|}{ Diabetic: } \\
\hline No overt retinopathy $(n=19)$ & $0.7(0.2)$ & $1.3(0.2)$ & $0.8(0.2)$ & $1.6(0.2)$ & $1.0(0.2)$ & $1.9(0.2)$ & $1.5(0.2)$ \\
\hline Vascular abnormalities $(n=6)$ & $0.5(0.3)$ & $0.8(0.4)$ & $0.3(0.3)$ & $1.2(0.5)$ & $1.1(0.4)$ & $1.7(0.4)$ & $1.3(0.4)$ \\
\hline $\operatorname{PDR}(n=6)$ & $1.0(0.5)$ & $1.8(0.4)$ & $1.5(0.4)$ & $1.8(0.3)$ & $2.2(0.3)$ & $2.0(0.4)$ & $1.8(0.4)$ \\
\hline Laser, no PDR (n=15) & $0.7(0.2)$ & $1.2(0.3)$ & $1.2(0.3)$ & $1.1(0.2)$ & $1.0(0.2)$ & $1.2(0.2)$ & $1.0(0.2)$ \\
\hline
\end{tabular}

$0=$ background, $1=$ weak, $2=$ moderate, $3=$ intense. 

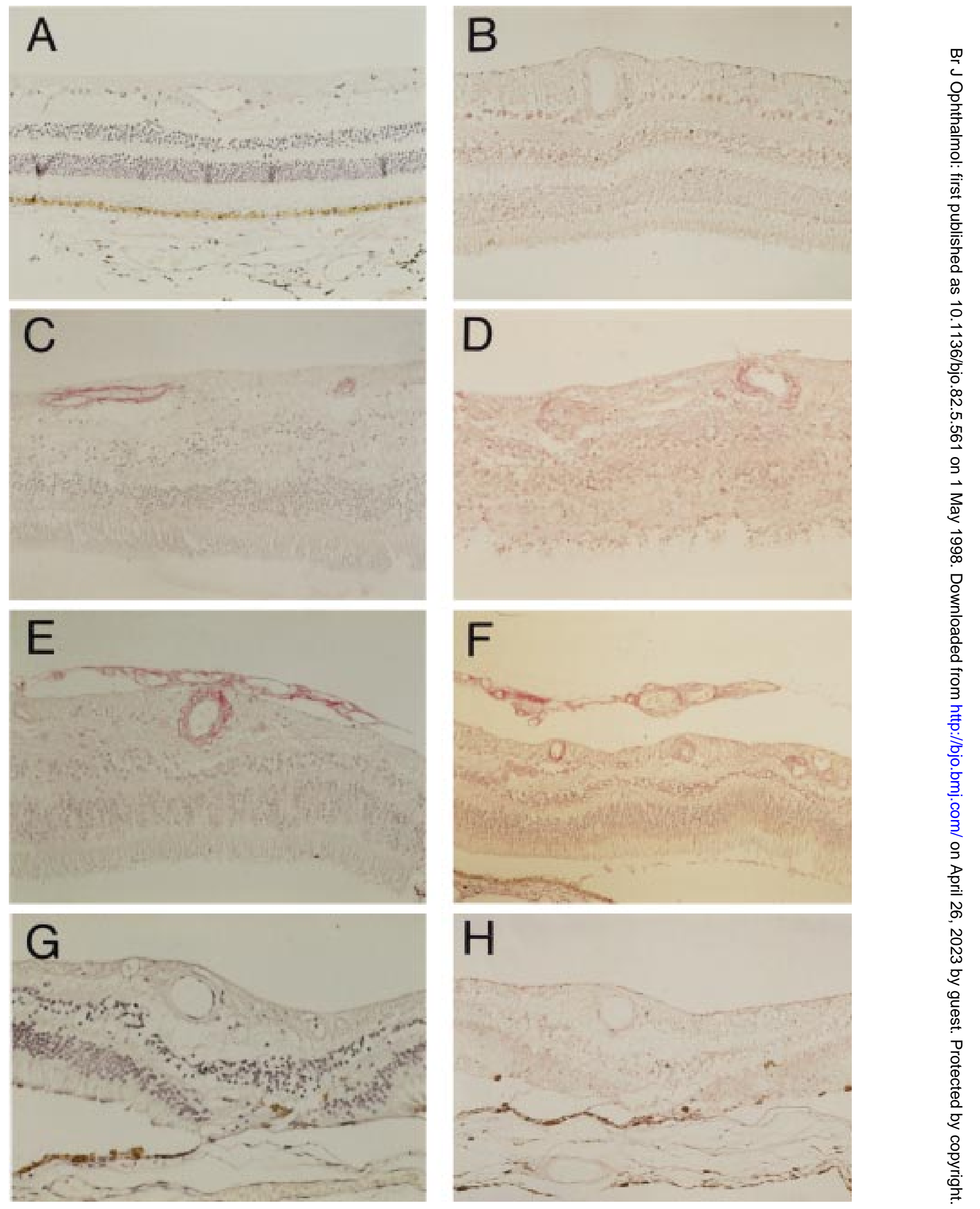

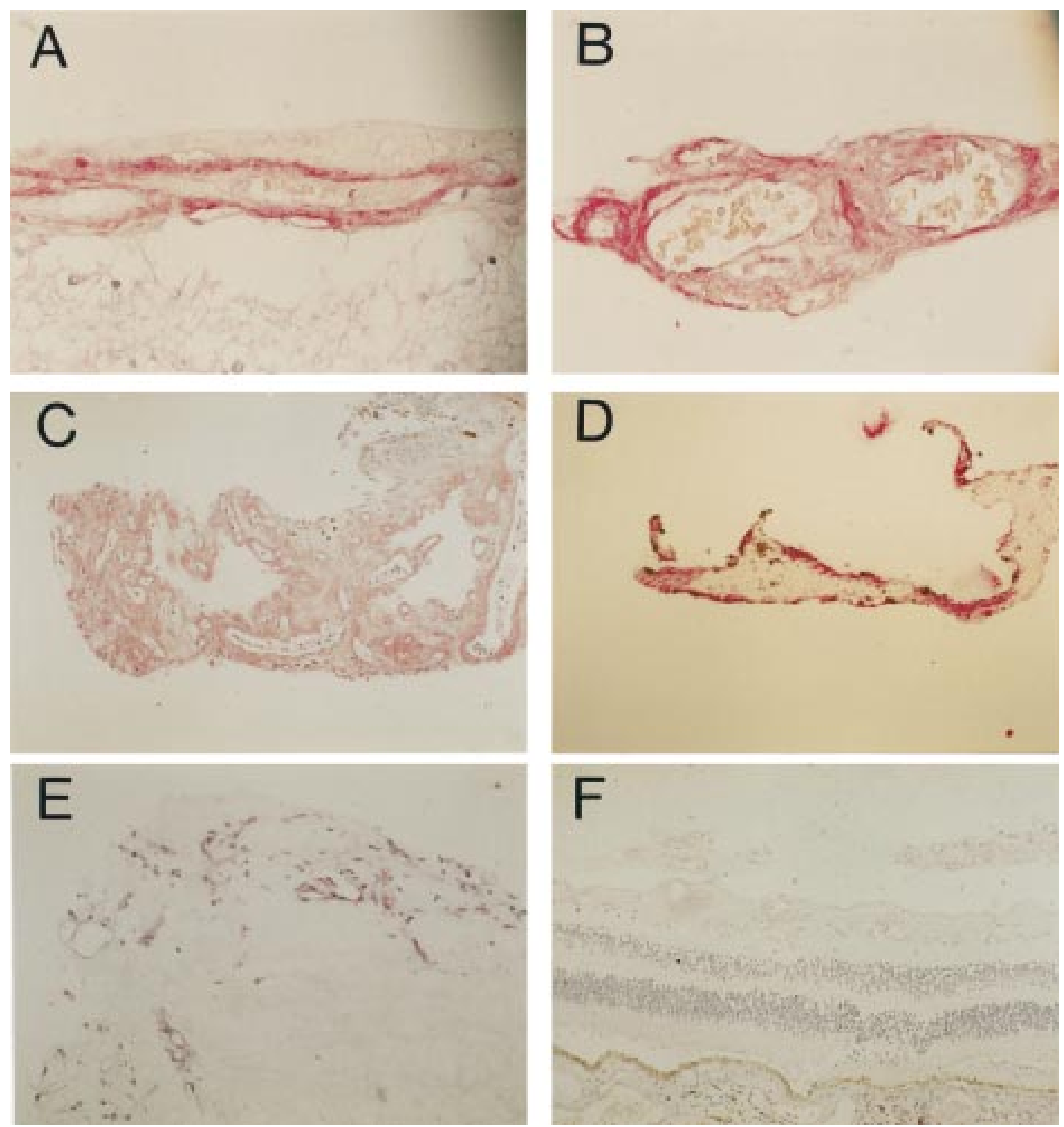

Figure 2 Photomicrographs demonstrating VEGF immunostaining of PDR retina and excised membranes. Intense immunostaining for VEGF $F_{165}$ is localised to the vasculature $(A)$ while VEGF staining can be observed in all specimens of excised fibrovascular (C) and fibrocellular (D) epiretinal membranes. Immunoreactivity for VEGF was abolished in control sections of PDR retina and membranes processed with omission of the primary antibody (E) or prior incubation of the antibody with VEGF Magnification, $A, B \times 200 ; C-F \times 70$.

No correlation was observed between VEGF positive glial staining and active neovascularisation.

DIABETIC WITH SCATTER LASER

PHOTOCOAGULATION BUT NO EVIDENCE OF PDR The intensity of immunostaining in diabetic retinas that had undergone apparently successful laser therapy (that is, those with no prereti-

nal neovascularisation) was similar to that observed for diabetic retinas with no overt retinopathy (Fig 1G, H). This was true for both antibodies used. In many laser treated retinas (11/15) a total absence of immunoreactivity was observed within inner retinal vessels, even those with thickened basement membranes. This was especially apparent in those vessels located near laser burns. Staining

Figure 1 Photomicrographs demonstrating VEGF immunostaining of normal retina $(A)$, diabetic retina with no obvious retinopathy (B), diabetic retina with obvious intraretinal vascular changes but no evidence of $P D R(C, D)$, diabetic retina with $P D R(E, F)$, and diabetic retina after laser treatment for $P D R(G, H)$. Sections were immunostained with either an antibody raised against VEGF $F_{165}(A, C, E, G)$ or VEGF was greatest in diabetic retinas with PDR for both antibodies tested, minimal in normal retinas, and intermediate in diabetic retinas without PDR. It was interesting to note that immunostaining in lasered diabetic retinas with no current evidence of PDR was greatly reduced compared with the staining intensity in retinas with PDR. Magnification, $A-E, G \times 90 ; F, H \times 70$. 
intensity was also reduced/absent in the outer nuclear layer, inner retina, ganglion cell layer, and ILM compared with diabetic retinas with PDR.

FIBROVASCULAR MEMBRANES

A similar staining pattern was observed for both anti-VEGF antibodies used. In general, two immunostaining profiles for VEGF were observed in the preretinal diabetic membranes; those in which staining was essentially confined to the vascular component $(9 / 19)$ and those in which moderate to intense staining was also found in areas of acellular matrix (10/19) (Fig 2B, C). Staining of vessels was variable both between and within specimens, with those vessels at the periphery generally staining more intensely than those in the centre of the membrane. In addition, staining of extracellular matrix was variable but also tended to be highest at the periphery. Staining of non-vascular cells was weak and variable, being present in only $7 / 19$ membranes.

FIBROCELLULAR MEMBRANES

VEGF staining was observed in all seven fibrocellular membranes; the staining profile was similar for both anti-VEGF antibodies. Staining was associated with pigmented cells, non-pigmented cells and surrounding matrix (Fig 2D). The intensity of staining was highly variable both within and between membranes and ranged from weak to intense. Intensity of staining in the matrix was generally greater than that found in the cellular areas.

CONTROLS

Immunoreactivity for VEGF was abolished in sections processed with omission or substitution of the primary antibody (Fig 2E) and considerably reduced by prior incubation of the antibody with recombinant $\mathrm{VEGF}_{165}$ (Fig $2 \mathrm{~F})$.

\section{Discussion}

We have demonstrated changes in the profile and intensity of VEGF staining dependent upon the severity of diabetic retinopathy. The most intense staining correlated with active neovascularisation and was present in both preretinal vessels and associated inner retinal vessels. Interestingly, the elevation of immunostaining for $\mathrm{VEGF}_{121,165,189}$ in diabetic retinas with no overt retinopathy infers that VEGF may play a role in both the preclinical and the proliferative stages of diabetic retinopathy.

Lutty and co-workers detected VEGF immunoreactivity within some smooth muscle cells of retinal arterioles and in the pericytes and some endothelial cells of retinal capillaries in non-diabetic human retinas. ${ }^{21}$ Furthermore, they noted a significantly increased immunoreactivity in the contractile elements of retinal vessels, in the endothelium of human diabetic retina and in and around the choroidal vessels, results consistent with those presented here. Immunohistochemical staining for VEGF has also been shown to be markedly increased in the retina of streptozotocin treated diabetic rats, with positive staining located in and around the inner retinal capillaries. ${ }^{22}$ However, in areas of non-perfused human diabetic retina and in rabbit and primate models of ocular angiogenesis, in situ hybridisation studies have shown hypoxia induced VEGF expression not in the component cells of retinal vessels but in the inner and outer nuclear layers or the ganglion cell layers. ${ }^{13}{ }^{14}$ This distribution is in agreement with immunostaining of VEGF within Muller cells and in the ganglion cell layer in oxygen induced neovascularisation models in rat retina ${ }^{2324}$ and in diabetic retina. ${ }^{25}$ We could only observe significant staining in these extravascular regions with one of the anti-VEGF antibodies used-that is, VEGF $_{121,165,189}$. This difference in specificity may reflect either (a) the availability of the appropriate epitope or (b) differential expression of VEGF isoforms within the retina. The generation of antibodies against the 121 and 189 VEGF isoforms may help address this question.

Rather than defining the sites of potential VEGF synthesis, the increase in staining for VEGF protein in diabetic retinal vessels demonstrated in our study, especially in eyes with neovascularisation, may reflect the localisation of sites of action or accumulation of the factor. Since VEGF binds to hypoxia inducible receptors found on endothelial cells, such cells surrounding non-perfused areas of diabetic inner retina may be likely to manifest increased VEGF binding. In addition, VEGF, especially the larger splice variants $\left(\right.$ VEGF $\left._{189,206}\right)$, binds to heparin ${ }^{26}$ and increased staining in and around retinal vessels may to some extent reflect binding to heparan sulphate associated with basement membrane thickening in diabetes.

We have demonstrated that VEGF protein increases in diabetic eyes and that staining is greatest in PDR. The role of VEGF in preclinical retinopathy is unclear but may relate to vascular permeability. Raised VEGF levels are known to raise vascular permeability, ${ }^{27-29}$ a feature prominent in background and preproliferative diabetic retinopathy. ${ }^{1}$ These observations suggest that VEGF is elevated in the diabetic retina in the absence of hypoxia and extensive retinal ischaemia inferring that other mechanisms (for example, hyperglycaemia) may upregulate VEGF. However, it should be noted that in this, as in other, ${ }^{25}$ studies it is difficult to determine the degree of retinal ischaemia, if any, in postmortem diabetic retinas with preproliferative retinopathy. While vascular permeability has been suggested as being a critical step in angiogenesis, ${ }^{30}$ VEGF itself is also likely to directly affect other cellular events associated with neovascularisation. ${ }^{31}{ }^{32}$ These observations are supported by Tolentino et al who reported that intravitreal injection of VEGF into primates resulted in leaky vessels, progressively dilated and tortuous vessels, microaneurysms, haemorrhage, and capillary closure. $^{33}$

Our observation that high levels of VEGF are associated with the periphery of preretinal vessels is further support that VEGF plays an 
important role in the progression of preretinal neovascularisation. The intense staining of acellular matrix in many of the membranes may also reflect the matrix binding properties of VEGF. VEGF present in PDR membranes may derive from the retina but may also be synthesised by the cells within the membrane. We observed staining for VEGF within non-vascular cells in some membranes, while mRNA for VEGF has been found in diabetic membranes. ${ }^{20}$ Vitreous fluid from patients with active retinal neovascularisation has previously been shown to contain high concentrations of VEGF. ${ }^{18}$ Independent of its origin, membrane and vitreous associated VEGF may propel the neovascular response resulting in membrane growth.

VEGF immunoreactivity in epiretinal membranes is in agreement with Chen and colleagues. $^{34}$ It is difficult to explain the presence of VEGF but it may reflect the hypoxic nature of this avascular tissue or that VEGF is modulating non-vascular cells; RPE cells $^{10}$ and fibrocellular membranes ${ }^{34}$ are known to express VEGF receptors.

Scatter laser photocoagulation induces regression of active diabetic neovascularisation. We have shown that immunostaining for VEGF is reduced in diabetic retinas that have no overt preretinal neovascularisation following laser therapy. This is in close agreement with Aiello et al who found decreased vitreous levels of VEGF in patients after laser therapy. ${ }^{18}$ It is possible that a reduction in retinal ischaemia after laser treatment reduces the production of VEGF suppressing neovascularisation and leading to regression and quiescence.

An expanding body of evidence, to which these results contribute, now suggests that VEGF may be Michaelson's ischaemia induced ocular angiogenic factor. However, it is unlikely that VEGF functions in isolation. Other angiogenic factor levels have been found to be elevated in diabetic retina, fibrovascular membranes, and vitreous including bFGF, IGF-1, TGF- $\beta$, PDGF, and placenta growth factor (PlGF). ${ }^{35}{ }^{36}$ Thus, VEGF may work synergistically with other factors; repeated intravitreal injection of VEGF into primates produces vascular abnormalities associated only with background retinopathy ${ }^{33}$ inferring that additional growth factors are required to initiate preretinal neovascularisation. Furthermore, VEGF may play a largely ignored, but important, role in modifying vascular permeability during the early stages of preproliferative retinopathy. Studies which determine the biological effects of VEGF and its interactions with other growth factors should increase our understanding of the vasoproliferative retinopathies and provide opportunities for new therapeutic interventions for these blinding conditions.

This work was supported by the British Diabetic Association, the Guide Dogs for the Blind Association, the Wellcome the Guide Dogs for the Blind Association, the Wellcome
Trust, and the Manchester Royal Eye Hospital Endowment Fund.
1 Garner A. Vascular diseases. In: Garner A, Klintworth GK, eds. Pathobiology of ocular disease. A dynamic approach. 2nd eds. Pathobiology of ocular disease. A dynamic approa

2 Michaelson IC. The mode of development of the vascular system of the retina with some observations on its significance for certain retinal disorders. Trans Ophthalmol Soc UK 1948;68:137-80.

3 Hanneken A, de Juan E, Lutty GA, et al. Altered distribution of basic fibroblast growth factor in diabetic retinopathy. Arch Ophthalmol 1991;109:1005-11.

4 Robbins SG, Mixon RN, Wilson DJ, et al. Platelet-derived growth factor ligands and receptors immunolocalized in
proliferative retinal disorders. Invest Ophthalmol Vis Sci proliferative retinal

5 Dills DG, Moss SE, Klein R, et al. Association of elevated IGF-1 levels with increased retinopathy in late-onset diabetes. Diabetes 1991;40:1725-30.

6 Minchenko A, Bauer T, Salceda S, et al. Hypoxic stimulation of vascular endothelial growth factor expression in vivo and in vitro. Lab Invest 1994;71:374-9.

$7 \mathrm{deVries} \mathrm{C}$, Escobedo JA, Ueno H, et al. The fms-like tyrosine kinase, a receptor for vascular endothelial growth factor. Science 1992;255:989-91.

8 Terman BI, Vermazen MD, Carrion ME, et al. Identification of the KDR tyrosine kinase as a receptor for vascular endothelial growth factor. Biochem Biophys Res Commun 1992;34:1578-86.

9 Quinn T, Peter KG, deVries C, et al. Fetal liver kinase 1 is a receptor for vascular endothelial growth factor and is selectively expressed in vascular endothelium. Proc Natl Acad Sci USA 1993;90:7533-7.

10 Guerrin M, Moukadiri H, Chollet P, et al. Vasculotropin/ vascular endothelial growth factor is an autocrine growth factor for human retinal pigment epithelial cells cultured in vitro. $\mathcal{F}$ Cell Physiol 1995;164:385-94.

11 Thieme H, Aiello LP, Takagi H, et al. Comparative analysis of vascular endothelial growth factor receptors on retinal and aortic vascular endothelial cells. Diabetes 1995;44:98103.

12 Tuder RM, Flook BE, Voelkel NF. Increased gene expression for VEGF and the VEGF receptor KDR/Flk and Flt in lungs exposed to acute or to chronic hypoxia. $\mathcal{f}$ Clin Invest 1995;95;1798-807.

13 Miller JW, Adamis AP, Shima DT, et al. Vascular endothelial growth factor/vascular permeability factor is temporally and spatially correlated with ocular angogenesis in a primate model. Am F Path 1994;145:574-84.

4 Pe'er J, Shweiki D, Itin A, Hemo I, et al. Hypoxia-induced expression of vascular endothelial growth factor by retinal cells is a common factor in neovascularising ocular diseases. Lab Invest 1995;72:638-45.

15 Pe'er J, Folberg R, Itin A, et al. Upregulated expression of vascular endothelial growth factor in proliferative diabetic retinopathy. Br f Ophthalmol 1996;80:241-5.

16 Stone J, Pe'er J, Chan-Ling T, et al. The roles of vascular endothelial growth factor and neuroglia in the pathogensis of retinopathy of prematurity. Invest Ophthalmol Vis Sci 1995;36(suppl):871

17 Pierce E, Foley ED, Smith LEH. Regulation of retinal vascular endothelial growth factor (VEGF/VPF) levels by hyperoxia and hypoxia: a possible etiology for ROP. Invest Ophthalmol Vis Sci 1995;36(suppl):871.

18 Aiello LP, Avery RL, Arrigg PG, et al. Vascular endothelial growth factor in ocular fluid of patients with diabetic retinopathy and other retinal disorders. N Engl f Med 1994;331: 1480-7.

19 Adamis AP, Miller JW, Bernal M-T, et al. Increased vascular endothelial growth factor levels in the vitreous of eyes with proliferative diabetic retinopathy. Am $\mathcal{F}$ Ophthalmol 1994; 118:445-50

20 Malecaze F, Clamens S, Simorre-Pinatel V, et al. Detection of vascular endothelial growth factor messenger RNA and vascular endothelial growth factor-like activity in proliferative diabetic retinopathy. Arch Ophthalmol 1994;112:147682 .

21 Lutty GA, McLeod DS, Merges C, et al. Localization of vascular endothelial growth factor in human retina and vascular endothelial growth factor in humal. Arch Ophthalmol 1996;114:971-7.

22 Murata T, Isibashi T, Khalil M, et al. Vascular endothelial growth factor plays a role in hyperpermeability of diabetic retinal vessels. Ophthalmic Res 1995;27:48-52.

23 Robbins SG, Penn JS, Conaway JR, et al. Distribution of vascular endothelial growth factor (VEGF) in normal and oxygen-injured rat retinas. Invest Ophthalmol Vis Sci 1995;36(suppl):871.

24 Youssri AI, Luma J, Vinores S, et al. Immunohistochemical localisation of vascular endothelial growth factor (VEGF) in retinas with oxygen-induced ischemic retinopathy and non-ischemic retinas. Invest Ophthalmol Vis Sci 1995; 36(suppl): 401 .

25 Amin R, Frank R, Kennedy A, et al. Vascular endothelial growth factor is present in glial cells of the retina and optic nerve of human subjects with non-proliferative diabetic retinopathy. Invest Ophthalmol Vis Sci 1997;38:36-47.

26 Houck KA, Ferrara N, Winer J, et al. The vascular endothelial growth factor family: four molecular species and characterisation of alternative splicing of RNA. Mol Endocrinol 1991;5:1806-14.

27 Senger DR, Van De Walter L, Brown LF, et al. Vascular permeability factor (VPF, VEGF) in tumor biology. Cancer Metastasis Rev 1993;12:303-24. 
28 Connolly DT, Olander JV, Heuvelman D, et al. Human vascular permeability factor. F Biol Chem 1989;264:20017-24.

29 Murata T, Nakagawa K, Khalil A, et al. The relation between expression of vascular endothelial growth factor and breakdown of the blood retinal barrier in diabetic rat retinas. Lab Invest 1996;74:819-25..

30 Dvorak HF. Tumors: wounds that do not heal. Similarity between stroma generation and wound healing. $N$ Engl $\mathscr{F}$ Med 1986;315:1650-6.

31 Ferrara N, Henzel WJ. Pituitary follicular cells secrete a novel heparin-binding growth factor specific for vascular endothelial cells. Biochem Biophys Res Commun 1989;161:851-9.

32 Leung DW, Cachianes G, Kuang W-J, et al. Vascular endothelial growth factor is a secreted angiogenic mitogen. Science 1989;246:1306-9.
33 Tolentino M, Miller J, Gragoudas E, et al Intravitreous injections of vascular endothelial growth factor produce retinal ischaemia and microangiopathy in an adult primate. Ophthalmology 1996;103:1820-8.

34 Chen Y-S, Hackett S, Schoenfeld C-L, et al. Localisation of vascular endothelial growth factor and its receptors to cells of vacular and avascular epiretinal membranes. $\mathrm{Br} F$ Ophthalmol 1997;81:919-26.

35 Boulton ME, Foreman D, McLeod D. Vascularised vitreoretinopathy: the role of growth factors. Eye 1996;10: 691-6.

36 Khaliq A, Foreman D, Ahmed A, et al. Increased expression of placenta growth factor (PlGF) in proliferative diabetic retinopathy. Lab Invest 1998; (in press). 